\title{
Sustentabilidad urbana como planificación estratégica
}

\begin{abstract}
From its origins in the Brundtland report, sustainable development was projected as an activity that was best generated and most appropriate at the local scale. This localisation of the phenomenon was also central to the thinking behind Local Agenda 21. Despite numerous worldwide initiatives along the lines of LA21, it is clear that local applications in metropolitan areas operating without an overarching city-wide framework oriented towards sustainability are likely to confront serious obstacles. While recognising the value of localised initiatives, it is vital that sustainability is at the core of metropolitan level planning. Specifically, the role of strategic urban planning has considerable overlaps with the pretensions of the sustainability agenda, such as high levels of participation, long time horizons, and integrated rather than sectoral decision-making. This article highlights these overlaps and the potentialities for regarding urban sustainability as a conceptual platform for strategic urban planning. Various metropolitan experiences of strategic planning are considered, particularly the case of Santiago de Chile.
\end{abstract}

Keywords: sustainable development, urban sustainability, strategic planning, Santiago de Chile.

\section{Resumen}

Desde sus orígenes en el informe Brundtland, el desarrollo sustentable era proyectado como una actividad más apropiada y más efectiva a escala local. Esta localización del fenómeno era central al pensamiento del Programa Local 21. A pesar de múltiples iniciativas alrededor del mundo, es claro que las aplicaciones locales en áreas metropolitanas que operan sin un marco orientado hacia la sustentabilidad a escala de la ciudad se verán probablemente enfrentadas con serios obstáculos. Sin disminuir la importancia de iniciativas locales, es clave que la sustentabilidad se establece en el corazón de la planificación de escala metropolitana. Más específicamente, el rol de la planificación urbana estratégica tiene traslapes importantes con las pretensiones de la agenda de sustentabilidad, como los altos niveles de participación, horizontes de largo plazo y toma de decisiones en forma integrada más que sectorialmente. Este artículo enfatiza estos traslapes y las potencialidades para entender la sustentabilidad urbana como una plataforma conceptual para la planificación urbana estratégica. Varias experiencias de planificación estratégica metropolitana están consideradas, en particular el caso de Santiago de Chile.

Palabras clave: desarrollo sustentable, sustentabilidad urbana, planificación estratégica, Santiago de Chile. 


\section{Un espacio para la planificación estratégica}

$\mathrm{L}$ a asociación entre la planificación urbana y la planificación de desarrollo estatal ha tenido un impacto significativo sobre la planificación durante el último cuarto del siglo veinte. La tendencia de favorecer una perspectiva menos estratégica de la planificación sobre horizontes de tiempo más largos, en favor de un proceso más parcial de conjuntos de proyectos e intervenciones sectoriales, ha tenido claras implicaciones para el desarrollo urbano. Tal vez el ejemplo más evidente de esta reformulación en el enfoque hacia el crecimiento urbano se ha producido en el discurso reflejado en la praxis: la planificación era reemplazada por la gestión en forma discursiva y en la práctica. El acento estaba puesto en la gestión de diversas intervenciones -a menudo a través de la micro-gestión por instrumentos normativos y financieros-con mucho menos énfasis en la planificación de futuros urbanos. En este sentido, la escala temporal es significativa. La gestión era generalmente una actividad de corto plazo, basada en administraciones de más o menos cinco años de duración. Por otro lado, la planificación urbana tiene implicaciones generalmente para un mínimo de cinco años, en términos de poner en marcha programas y actividades que establecen una plataforma más estable para el desarrollo futuro; un plan de cinco años tiene pocas posibilidades de establecerse y tener frutos antes del quinquenio siguiente. Este paradigma de gestión urbana, que reemplazó al paradigma de planificación en muchas administraciones desde los años ' 70 , ha sido claramente asociado con el auge del neoliberalismo.

La tendencia hacia el neoliberalismo, asociada con la emergencia de la "nueva derecha" en Estados Unidos (por ejemplo la administración de Reagan) y Europa (en particular la administración de Thatcher), así como también en América Latina (la dictadura de Pinochet y los regímenes autoritarios de Brasil y de Uruguay) y otras regiones, fue dramática y amplia en sus impactos. Las privatizaciones de servicios públicos, desarrollo infraestructural y provisión de vivienda fueron típicos de esta tendencia.

Instituto de Estudios Urbanos y Territoriales, Pontificia Universidad Católica de Chile. E-mail: jbarton@puc.cl
No obstante, el nuevo siglo trajo una ola de escepticismo frente a la capacidad de realizar un desarrollo urbano más comprensivo e integrado basado en este modelo neoliberal de determinaciones de mercado. Bajo éste, el rol del Estado fue relegado a facilitador y regulador (normalmente más eficaz en el primero, altamente ineficaz en el segundo). Frente a esta situación, el nuevo discurso que surgió desde fines de los años ' 90 postuló un rol menos facilitador y más organizacional por parte del Estado. Este discurso se puede definir como "neoestructural".

Asociado con esta tendencia neoestructural en la planificación, el Estado retoma algunas de las funciones que fueron tan centrales a sus actividades en el período que se entiende desde la posguerra hasta fines de los años '70, y las vincula con las formulaciones más contemporáneas de la planificación, como se puede ver en el sector privado ${ }^{1}$. La planificación estratégica es un instrumento que une estas (re)formulaciones. Se puede ver, por ejemplo en el plan estratégico de Madrid de 2003, donde se hace el contraste entre la planificación urbana tradicional y la planificación estratégica. Las tres características principales contrastadas son: a) la mayor flexibilidad de la planificación estratégica, con la posibilidad de modificar los objetivos próximos para alcanzar los objetivos últimos claves; b) el enfoque en mejorar la competitividad económica y la calidad de vida más que ordenar el uso de suelo y la infraestructura, yc) la centralidad de la participación y la construcción de consenso (ProMadrid S.A., 2003).

Referente la primera característica y su openendedness (o no-lineariedad y su proceso de aprendizaje asociado [Hjorth y Bagheri, 2006]), se puede incorporar la perspectiva sobre el desarrollo sustentable ofrecido por Meadowcroft (1999: 37): "El desarrollo sustentable es mejor entendido como un 'meta-objetivo' social de largo plazo, y como una línea base idealista con la cual evaluar las prácticas

\footnotetext{
Hay varias formas de planificación estratégica que se usan en actividades del sector privado, como el Modelo Harvard, los Sistemas de Planificación Estratégica, los Enfoques de Gestión de Stakeholders, los Enfoques de Contenido, la Gestión de Temas Estratégicos y las Estrategias de Proceso. En la documentación de estos ejemplos, Bryson y Roering (1993) observan que en su traducción hacia el sector público, es más probable que se hibridicen debido a los factores únicos de cada contexto de planificación.
} 
contemporáneas. Es el resultado que prosigue cuando el desarrollo genuino se mantiene dentro de las fronteras de la prudencia ambiental, también un estándar normativo exigente que requiere el equilibrio entre medio ambiente, actividad económica y equidad social en la toma de decisiones actuales. Por ende, el desarrollo sustentable es 'sin fin' (openended)".

La planificación urbana estratégica orientada hacia el paradigma de la "sustentabilidad como proceso" es una construcción en esta línea (referida en adelante como la "planificación estratégica sustentable”). Es neoestructural, debido a la centralidad del Estado a través de la promoción y seguimiento de metas de largo plazo, dirigido por agencias públicas que funcionan como un pivote para la incorporación de una amplia base de stakeholders ${ }^{2}$. Los componentes "neo" se relacionan con muchas de las innovaciones en planificación que han surgido en conjunto con -o en oposición a- la gestión urbana neoliberal. Otros componentes asociados con la orientación neoliberal de la gestión urbana se relacionan principalmente con el involucramiento del sector privado en diversas áreas -desde vivienda a vialidad, cárceles a almuerzos escolares, agua a telecomunicaciones. De importancia específica han sido los mecanismos financieros para facilitar estas iniciativas público-privadas. Las concesiones, subcontrataciones a través de licitaciones, venta de entidades públicas, subasta de frecuencias de transmisiones y otras formas de asociación han evolucionado. Se pueden agregar a estos mecanismos varias tendencias contra-cíclicas que han surgido en oposición a esta asociatividad público-privada, y relacionadas con cambios de gran envergadura a nivel de la sociedad civil desde principios de los años ' 80 . Entre ellos, enfatizo la importancia de temas ambientales, como también los de raza y etnicidad, el desarrollo social respecto a la violencia, delincuencia y drogas, la rehabilitación de sitios "cafés" e históricos, participación de la sociedad

Riddell (2004) favorece el término "neomoderno" para los estilos de vida equilibrados social, ecológica y económicamente, que deben reemplazar los estilos de vida modernos y no-sustentables. Este sería el objetivo de este enfoque, más que los medios neoestructurales. civil en la toma de decisiones y movimientos sociales enfocados en "problemas" urbanos ${ }^{3}$.

La combinación de temas tradicionales y más contemporáneos, como también la reformulación de las estructuras institucionales, constituye el centro del pensamiento urbano neoestructural. Los límites de la urbanización de "comando y control" y también los de la gestión urbana neoliberal son reconocidos, y nuevas opciones han presentados para el debate y el desarrollo. Entre ellas, se puede argumentar que la planificación urbana estratégica basada en los principios de la sustentabilidad tiene el potencial para ser la más significativa. Como Ache (2000) documenta, en el contexto de las ciudades de Renania del Norte-Westfalia, un enfoque estratégico de las ciudades-regiones basado en visiones y creatividad (a través del desarrollo de información, comunicaciones y conocimiento) es necesario para transformar las ciudades en agentes de cambio positivo.

\section{La planificación estratégica urbana: grados de sustentabilidad}

Es importante en este momento contrastar la planificación estratégica sustentable con la planificación estratégica basada en los principios de la gestión urbana neoliberal. Son diferentes en el sentido que la primera ofrece una perspectiva integrada de largo plazo del desarrollo urbano, en tanto que la segunda enfatiza en los objetivos de corto plazo del crecimiento urbano. Este crecimiento es comprendido en términos económicos, medido por el PIB en particular, más un conjunto de otras variables asociadas como el empleo, valores de suelo e inversiones. Se manifiesta más comúnmente a través del crecimiento urbano morfológico, como el sprawl de la expansión dirigido por el mercado del área urbana en espacios anteriormente rurales, por ciudades satélites, ciudades dormitorios o edge cities.

Sería ingenuo subestimar el rol del crecimiento económico en el desarrollo urbano. El motor de la economía -con los combustibles de flujos de capital

Estos son "problemas" de diversas índole, desde "NIMBYs" (Not In My Back Yard) a "LULUs" (Locally Unwanted Land Use), referentes a nueva infraestructura, temas de identidad y tolerancia y respuestas a altos niveles de criminalización, entre otros. 
y la fuerza laboral- es lo que hace funcionar la ciudad. No obstante, el modelo de desarrollo urbano -que sugiere que los desafíos del desarrollo urbano son efectivamente resueltos por el crecimiento- es precisamente el modelo de desarrollo que ha sido criticado más durante el último cuarto del siglo veinte, cuando estaba en su cenit ${ }^{4}$. Bajo este período, el motor estaba andando pero no fue claro hacia dónde se dirigía el vehículo, ni el grado de confort de los pasajeros. La velocidad (crecimiento en PIB) del vehículo apareció como el único factor importante. La teoría sobre modernización de Rostow (1960) y su "efecto goteo" para conseguir la "era de consumo masivo" ha sido ampliamente criticada (Wallerstein, 1974; Escobar, 1995), así como también el modelo de desarrollo neoliberal impulsado por el Consenso de Washington (Stiglitz, 2002).

La planificación sustentable ofrece una perspectiva más equilibrada y transversal de este crecimiento. La calidad del crecimiento es enfatizada sobre la cantidad del crecimiento 5 . La pregunta que surge es: ¿qué tipo de crecimiento se necesita para alcanzar los mejoramientos en la calidad de vida de la sociedad en el largo plazo? Esta pregunta -y sus variantes- son centrales para la planificación sustentable. La construcción destacada por Turner (1992) de la sustentabilidad débil hasta la sustentabilidad fuerte es útil en este sentido, en términos de concretar la lógica de la sustentabilidad en un contexto de planificación. La base de esta diferenciación conceptual es la capacidad de sustitución entre distintos tipos de capitales: hecho por humanos $(\mathrm{Km})$; natural $(\mathrm{Kn})$; humano $(\mathrm{Kh})$; ético y moral $(\mathrm{Ke})$ y cultural $(\mathrm{Kc})$, según cuatro posicio-

4 Los disturbios y desórdenes motivados por conflictos raciales, pobreza y delincuencia (también interrelacionados en muchos casos) en ciudades percibidas como altamente desarrolladas en contextos nacionales e internacionales, ejemplifican los límites de esta forma de pensar -por ejemplo París (octubre-noviembre de 2005) y São Paulo (mayo de 2006).

Existe un paralelo con "la democracia". En América Latina, gran parte del debate durante los años '80 hasta la mitad de los '90 se centraba en el retorno de la democracia. Esta discusión, en años más recientes, se ha transformado y está orientada hacia la calidad de la democracia y su capacidad de realizar diversas aspiraciones (Tedesco y Barton, 2004). Los datos de Latinobarómetro identifican la reducción en el apoyo hacia las nuevas democracias en la región debido a sus debilidades en realizar las ambiciones de la sociedad en su forma más amplia. nes: muy débil, débil, fuerte y muy fuerte. La sustentabilidad muy débil asume que existe una sustitución perfecta entre el capital natural $(\mathrm{Kn})$ y el capital hecho por humanos $(\mathrm{Km})$, con el objetivo de mantener el stock total de capital (capital constante), mientras que la sustentabilidad muy fuerte asume una economía estable (steady-state).

Un modelo intermedio de "sustentabilidad fuerte", favorecido por el autor, se orienta hacia el objetivo de la posición de los activos de capital constante, basado en un enfoque precautorio hacia la base de capital natural crítico (de recursos naturales renovables y no-renovables y calidad ambiental), que toma en cuenta las dimensiones de incertidumbre, irreversibilidad e insubstitutability de esta base. Esta perspectiva de sustitución de capitales es relevante para la planificación estratégica sustentable, en el sentido que identifica el rango de variables que hay que tomar en cuenta y sus relaciones con otras variables. En la misma línea, Riddell (2004) anota que la sustentabilidad tiene sus orígenes políticos y sociales en un estilo de "bancar recursos", que sería una metáfora apropiada para este enfoque de capitales frente a la sustentabilidad.

Es importante enfatizar la variable del capital ético y moral $(\mathrm{Ke})$, que a menudo no está muy presente en el proceso de planificación, a pesar de su relevancia para el principio de equidad de la sustentabilidad $^{6}$. En el caso de la planificación metropolitana, es necesario responder a lo que se estaba destacado-pero inadecuadamente desarrollado-en el Plan de Implementación de Johannesburgo en 2002 (Introducción, artículo 6; CSD, 2002, 3): "Reconocemos la importancia de la ética para el desarrollo sostenible y, por consiguiente, subrayamos la necesidad de tenerla en cuenta en la aplicación del Programa 21".

\section{La praxis de la sustentabilidad}

A pesar de los debates relacionados con definiciones y las implicaciones prácticas del pensamiento sobre el desarrollo sustentable, es evidente que el

6 Es interesante anotar que la introducción escrita por el Intendente a la Estrategia de Desarrollo Regional para Santiago de Chile 2000-2006 termina con un énfasis sobre el "imperativo ético" del desarrollo regional metropolitano, a la luz del gran número de familias pobres viviendo en la región (Gobierno de Chile, 2000). 
concepto ha sido un código importante para la organización del desarrollo nacional, regional, rural y urbano a través de distintos continentes, países y ciudades. Se puede ver, por ejemplo, en el caso de los compromisos de Aarlborg de la Campaña de las Ciudades Europeas Sostenibles en 1994, en 1996 con la Declaración de Santa Cruz de la Sierra de los ministros de medio ambiente de la OEA, y más recientemente, en la Declaración de Hong Kong de 2005 firmada por representantes de ciudades de Asia.

Aunque el informe de la Comisión Mundial sobre Medio Ambiente y Desarrollo (1987) -mejor conocido como el informe Brundtland-fue explícito aclarando sus consideraciones sobre los desafíos para las sociedades contemporáneas, no fue hasta 1992 que el Programa 21 indicó posibles programas y actividades para alcanzar lo que el primero presentaba como requisitos para el desarrollo sustentable (véase Cuadro 1). El informe mostró un marco amplio de sistemas interconectados, cada uno con una clara orientación, mientras que los criterios del Programa 21 indicaron los aspectos de gestión a nivel local. Como se puede ver en el cuadro, hay claridad respeto a los objetivos y las dimensiones de proceso (incluyendo evaluación). Los medios específicos también son presentados en los sectores de implementación del programa. La selección de cada instrumento y cuadros interconectados de instrumentos es explícitamente definida por los tomadores de decisiones a nivel local, a la luz de las prioridades locales, consideraciones de recursos, trade-offs y conflictos (véase Gibson et al., 2005).

El Programa 21 ha ofrecido un marco amplio de objetivos y actividades que se puede activar a nivel local. No obstante, el éxito con el cual el Programa 21 ha sido utilizado en los niveles nacionales y locales es cuestionable. De todas maneras, a pesar de un lento despegue durante los años ' 90 , es cierto que más municipalidades manejan actividades que cumplen con los criterios actualmente definidos por la Comisión de Desarrollo Sostenible (CSD) e ICLEI (véase Cuadro 1). Si se combinan los requisitos identificados en el informe Brundtland con los criterios de la CSD/ICLEI para la evaluación de las actividades del Programa 21 alrededor del mundo, es posible definir los principios claves o criterios para el desarrollo sustentable, y entonces para la planificación sustentable estratégica. Todos estos principios son aplicables a los espacios urbanos, y el desafío para las ciudades más grandes es que están más claramente en los "intersticios" de las tendencias globalizadas y localizadas (para usar el término de Swyngedouw (1992), en los 'intersticios de la globalización”; véase también Marcotullio, 2001).

Si se agregan al Programa 21 (que fue reiterado en la Cumbre de Johannesburgo) las Metas del Milenio surgidas en la Cumbre de Desarrollo Social en Copenhague en 1995 y ratificadas por todos los miembros de la ONU en el año 2000 en Nueva York, se puede observar la naturaleza transversal de la agenda de sustentabilidad establecida a nivel multilateral. No obstante, no es solamente a nivel multilateral que estas agendas, metas y objetivos se quedan. Es a través de su aplicación a niveles nacionales, regionales y locales que se consigue el desarrollo sustentable más fuerte. Es este aterrizaje del concepto a nivel local que se puede entender la praxis de la sustentabilidad.

La agenda de sustentabilidad asociada con el Programa 21 siempre ha sido enfocada a nivel local; el término Agenda Local 21 (LA21) surgió para enfatizar este punto, aunque el título del plan general (o blueprint) presentado en Río de Janeiro en 1992 era simplemente Programa 21 (Agenda 21). El tono del documento está de todas maneras orientado hacia la escala local. Esto es particularmente relevante para las áreas metropolitanas que pueden agrupar varias municipalidades ${ }^{7}$. Claramente existe un ámbito considerable para integrar las estrategias municipales más locales dentro de un marco orientador que incorpore la ciudad-región. Esto ha sido el objetivo de la planificación estratégica en general para Bilbao, Vancouver, Santiago de Chile y otros. En este sentido, es interesante comparar los criterios para la planificación estratégica urbana (Cuadro 2) establecidos por Fernández Güell (2000), con los de la agenda de la sustentabilidad (Cuadro 1).

Es evidente que los objetivos más amplios a niveles institucionales y espacios superiores son nece-

Parece un sinsentido el que una municipalidad adopte un plan de sustentabilidad que enfatiza emisiones bajas de vehículos privados y trabaja hacia este fin, si el municipio vecino enfatiza la accesibilidad del vehículo privado sobre el transporte público a través de nueva infraestructura vial. 
Cuadro 1. Los Criterios de la Sustentabilidad.

\begin{tabular}{|c|c|}
\hline WCED (1987) & CSD-ICLEI $(1996,2002)$ \\
\hline Requisitos de una Estrategia Sustentable & Criterios de Evaluación del Programa 21 \\
\hline $\begin{array}{l}\text { Un sistema político que asegura la participación efectiva de } \\
\text { los ciudadanos en la toma de decisiones }\end{array}$ & $\begin{array}{l}\text { Debe enfrentar las necesidades económicas, sociales y } \\
\text { ecológicas en forma conjunta }\end{array}$ \\
\hline $\begin{array}{l}\text { Un sistema económico que puede generar superávit y cono- } \\
\text { cimiento técnico en una forma auto-suficiente y sostenida }\end{array}$ & $\begin{array}{l}\text { Debe incluir un consenso sobre una visión para un futu- } \\
\text { ro sustentable }\end{array}$ \\
\hline $\begin{array}{l}\text { Un sistema social que ofrece soluciones para las tensiones } \\
\text { que surgen desde el desarrollo no-armónico }\end{array}$ & Debe incluir un proceso participativo con residentes locales \\
\hline $\begin{array}{l}\text { Un sistema de producción que respeta la obligación a } \\
\text { preservar la base ecológica del desarrollo }\end{array}$ & $\begin{array}{l}\text { Debe establecer un Grupo de Stakeholders, Foro o un } \\
\text { grupo comunitario multisectorial equivalente para vigilar } \\
\text { el proceso }\end{array}$ \\
\hline $\begin{array}{l}\text { Un sistema tecnológico que puede buscar en forma cons- } \\
\text { tante nuevas soluciones }\end{array}$ & Debe preparar un Plan de Acción \\
\hline $\begin{array}{l}\text { Un sistema internacional que promueve padrones de co- } \\
\text { mercio y financiamiento sustentable }\end{array}$ & $\begin{array}{l}\text { Debe preparar un Plan de Acción con metas de largo } \\
\text { plazo }\end{array}$ \\
\hline $\begin{array}{l}\text { Un sistema administrativo que es flexible y tiene la capaci- } \\
\text { dad de autocorrección }\end{array}$ & Debe establecer un marco de monitoreo y evaluación \\
\hline- & Debe establecer indicadores para medir el progreso \\
\hline
\end{tabular}

Fuente: WCED (1987), CSD-ICLEI (2002).

Cuadro 2. Las Características de la Planificación Estratégica de Ciudades.

\begin{tabular}{|l|}
\hline - Visión a largo plazo y carácter plurilegislativo \\
\hline - Consideración del entorno \\
\hline - Identificación de la ventaja competitiva \\
\hline - Visión integral de la realidad urbana \\
\hline - Flexibilidad decisional \\
\hline - Concentración en temas críticos \\
\hline - Orientación a la acción \\
\hline - Fomento y coordinación de la participación de todos los agentes sociales \\
\hline - Modernidad de la administración \\
\hline Fuente: Fernández Güell (2000).
\end{tabular}

Fuente: Fernández Güell (2000).

sarios. Efectivamente es el caso más común, pero solamente a nivel sectorial: por ejemplo, estrategias de aire limpio, facilidades para el tratamiento de agua, estrategias de gestión de residuos sólidos, políticas de empleo y de transporte. El techo que fijan estos pilares sectoriales para proveer una edificación sólida es, lamentablemente, raramente construido. Los componentes interconectados que trascienden las intervenciones y políticas sectoriales son débiles como consecuencia, y no encuentran espacio dentro de las arquitecturas institucionales o dentro de los instrumentos de planificación existentes. Tal vez esta debilidad se puede explicar debido al significado y rol potencialmente poderoso de los componentes interconectados en la orientación del desarrollo urbano. A través del control del marco dentro del cual las intervenciones sectoriales e intereses se encuentran, ofrece a sus diseñadores y gerentes una base de poder considerable con la cual negociar y resolver conflictos sobre recursos, propiedad y desarrollo (Campbell, 2005). Es por esta misma razón que los ejercicios contemporáneos de planificación sustentable son lo más participativos posible, al menos en forma transversal en términos de las agencias públicas, y más probablemente en conjunto con un amplio rango de otros stakeholders (véase Allen et al., 2002; Gross, 2002; Unsworth, 2004). Más que un enfoque "experto" de "caja negra" hacia la planificación, radicado dentro de la autoridad gubernamental "responsable", es un enfoque de diseño por discusión, como charettes, grupos de expertos y no-expertos o grupos de stakeholders bien definidos.

Antes de detallar cómo estos factores entran en juego en las secciones siguientes, es relevante anotar el grado en que la agenda de sustentabilidad multilateral ha sido orientada hacia los espacios urbanos, y el grado en que discursivamente esta agenda ha sido adop- 
tada por instituciones nacionales, regionales y locales. Aunque el informe Brundtland -el producto de foros regionales e información experta entre 1983 y 1987- es reconocido como el documento principal respecto al desarrollo sustentable, sus raíces son más profundas. La conferencia de Estocolmo en 1972 y la creación de PNUMA, la primera conferencia de Hábitat en Vancouver en 1976, el informe de la Unión Internacional para la Conservación de la Naturaleza en 1980, los informes Brandt (1980 y 1983) y Hábitat II en Estambul (1996), entre otros, indicaron las debilidades del modelo de desarrollo dominante en la posguerra. El rol contemporáneo de los centros urbanos, en particular los centros urbanosindustriales, fue clave para estas discusiones; en Estocolmo, por ejemplo, dominaron las preocupaciones de la contaminación atmosférica (lluvia ácida; véase Hajer, 1997). Las consideraciones urbanas han persistido en objetivos e institucionalidad multilateral en los años siguientes. Eso se puede observar en la Cumbre de Johannesburgo (2002), las Metas del Milenio y los planes de trabajo de Hábitat, PNUMA y la Comisión de Desarrollo Sostenible (todos de la ONU).

En el caso de Hábitat, los vínculos entre la pobreza y el desarrollo urbano son destacados y estas preocupaciones han sido transferidas a las Metas, en particular bajo el tema ambiental (número 7), aumentando el acceso a agua potable, la erradicación de la vida en tugurios para 100 millones de personas y el objetivo menos claro de vincular políticas (UN, 2006): "Incorporar los principios del desarrollo sostenible en las políticas y los programas nacionales; invertir la pérdida de recursos del medio ambiente"s.

En forma más específica en términos de la sustentabilidad, la creación del Programa de Ciudades Sostenibles (SCP) de PNUMA-Hábitat en 1991 ha sido significativo en promover intervenciones, principalmente ambientales, en ciudades en América Latina, África y Asia y también en Rusia y Polonia. En América Latina la lista de 18 ciudades incluye San José (Costa Rica), Bogotá (Colombia), Quito

\footnotetext{
8 Las mediciones para esta primera parte de la Meta 9, la de integrar los principios del desarrollo sustentable en políticas y programas nacionales, están correlacionadas con cobertura forestal, áreas de protección de la biodiversidad, uso de energía y emisiones de dióxido de carbono.
}

(Ecuador), Lima-Callao (Perú), Concepción (Chile), Cienfuegos (Cuba) y Piranhas (Brasil). Este programa funciona en estrecha relación con los diagnósticos de las circunstancias ambientales en centros urbanos manejados por el programa GEO Ciudades de PNUMA. En la definición de presiones, del estado de las condiciones ambientales, de los impactos socioeconómicos y ambientales y de las posibles respuestas, el programa GEO Ciudades ha facilitado un proceso importante de recolección y evaluación de datos. La crítica principal (desde la perspectiva de la sustentabilidad) de los dos programas: SCP y GEO Ciudades es su orientación predominantemente ambiental. Ello varía un poco con los programas que poseen una plataforma más transversal como los de Hábitat y la CSD. En este contexto, se puede enfatizar el Plan de Implementación de la Cumbre de Johannesburgo con su identificación de los desafíos urbanos en términos amplios, en particular en la vivienda, el transporte, energía y servicios (UN, 2002).

Para América Latina, y Santiago de Chile en particular, se pueden agregar las iniciativas de orientación regional. La conferencia de los ministros de medio ambiente en Santa Sierra de la Cruz (Bolivia) en 1996 no solamente destacó la necesidad para las sociedades latinoamericanas de seguir un camino hacia estrategias de producción y consumo más sustentables, sino también aclaró los desafíos presentados por los altos niveles de urbanización en la región (II.3 del Plan de Acción) y la necesidad de incorporar la dimensión del desarrollo sustentable en la planificación urbana (Iniciativa 45) (OAS, 1996); una década más tarde, muchos de los mismos desafíos se han conservado (véase Winchester, 2005).

La iniciativa más reciente está relacionada con la decisión que surgió desde la Cumbre en Johannesburgo de formar una institución regional: la Iniciativa para América Latina y el Caribe de Desarrollo Sustentable de promover el Programa 21 y otros elementos en el Plan de Implementación. En el campo de ciudades más sustentables, se puede esperar un paso inicial muy parecido a la Declaración sobre el Desarrollo Sustentable de Ciudades, hecho por representantes urbanos en Asia en febrero 2004. La esencia de la Declaración está, tal vez, mejor expresada en el artículo 4: "En una era de 
globalización acelerada, las ciudades enfrentan desafíos y oportunidades. Más que nunca, las ciudades representan el nexo entre el comercio, la industria, finanzas, cultura, transporte y comunicación, con el potencial de conectar todas las áreas, rural y urbana, y todos los segmentos de la sociedad, en una fuerza productiva que conduce hacia una globalización y urbanización justa, equitativa y sustentable" (Asia and Pacific Leadership Forum, 2004).

Aunque se puede argumentar que la agenda del desarrollo sustentable tiene ambigüedades conceptuales y que el progreso ha sido lento en tomarla en cuenta como "mapa" para la planificación del desarrollo urbano, es claro que se han dado pasos significativos. En el Plan de Londres de 2004, por ejemplo, la sustentabiliidad fue un tema destacado, central no solamente para el plan de 15-20 en sí, sino también para iniciativas a lo largo de la ciudad que surgieron de la Greater London Authority.

Para explicar por qué algunas cuidadas han sido vanguardistas y otras más reticentes en la incorporación de los criterios de sustentabilidad a su planificación, debemos remitirnos a aspectos de conciencia, de gobernanza, de pensamiento visionario de líderes claves (principalmente en la administración pública), y también aludir a la sensibilidad frente a las presiones de la sociedad civil y la capacidad de respuesta frente a los compromisos multilaterales. No se debe olvidar que muchos países han firmado el acuerdo del Programa 21 y también las Metas del Milenio y por lo tanto, están "comprometidos" a realizarlos dentro de sus territorios. En la sección siguiente, algunos temas claves relacionados con la planificación estratégica sustentable-compromiso, orientación, objetivos últimos y próximos, temporalidad y participación-serán tratados.

\section{Comparando estrategias metropolitanas}

Los distintos grados de sustentabilidad en el discurso y la praxis se pueden analizar a través de las experiencias de la planificación estratégica metropolitana que existen. Es instructivo contrarrestar dos experiencias recientes de la planificación estratégica basadas en distintos enfoques: un caso de aparentemente fuerte de sustentabilidad, el de Vancouver y su Plan Estratégico de Región Vivible, y uno de sustentabilidad débil, el de Bilbao y su plan Bilbao Metrópoli- $30^{9}$.

En abril de 2001, Bilbao Metrópoli-30 juntó las treinta municipalidades de la ciudad dentro de un plan estratégico para 2010. Su orientación y objetivo principal fueron definidos bajo la visión de "desarrollar un contexto avanzado de conocimiento que propicie que las iniciativas empresariales altamente innovadoras se hagan realidad" (Bilbao Metrópoli 30, 2006). Dentro de esta estrategia de crear "una ciudad para el conocimiento y la innovación" se encuentran programas como la renovación urbana (del Casco Viejo), una exposición internacional y la planificación en torno de un río Bilbao más limpio. Tal vez el punto más importante para destacar en la experiencia Bilbao Metrópoli-30 sea la orientación económica de sus actividades. Esta experiencia es loable; sin embargo, el hecho de que el autoproclamado "Atractivo de la Ciudad" (uno de los pilares de la estrategia, con "Las Personas" y "La Actividad de la Ciudad"), sea actualmente el objetivo último de la estrategia, más que un objetivo próximo, es muy relevante: "ElAtractivo de la Ciudad: La Asociación Bilbao Metrópoli-30 parte de la consideración de la ciudad como un espacio vital, un lugar habitado y habitable, que tiene como objetivo prioritario lograr un medio donde el ser humano encuentre un ambiente favorable para su desarrollo armónico y solidario en lo personal y en lo social".

Si uno evalúa los vínculos entre el objetivo estratégico y estos tres pilares, en particular lo referente a "lo atractivo", no está claro cómo uno está sustentado o mejorado por el otro. Tal vez sería posible armar conexiones implícitas; sin embargo, la sugerencia que fundamenta la estrategia es que lo atractivo de la ciudad se vería aumentado por la nueva orientación económica. Al mismo tiempo, esta nueva orienta-

Son muchas las ciudades que empezaron a implementar la planificación estratégica desde principios de los años '80. Fernández Güell (2000) estima que la primera fue San Francisco en 1982, aunque se puede argumentar que otras la adelantaron a través de sus orientaciones implícitas de planificación estratégica, como Curitiba con su influyente Plan Maestro de 1966 (Macedo, 2004). Bilbao fue una de varias ciudades españolas que siguieron la planificación estratégica metropolitana, empezando con Barcelona en 1987 en su preparación para los Juegos Olímpicos. Otras incluyen Madrid, Jerez y Cádiz. 
ción económica parece depender de lo atractivo de la ciudad.

La planificación sustentable funciona sobre la premisa de que este atractivo es la raison d'être del proceso de planificación. Los otros elementos son instrumentos para la realización de esta condición. La referencia al "desarrollo armónico" es una alusión implícita a ello, aunque su significado no es explorado dentro de la estrategia. Es asumido como algo deseado producto de una economía competitiva dentro de una red de ciudades globales.

En contraste, la base para la estrategia metropolitana de Vancouver refleja los principios de la planificación para la sustentabilidad fuerte. En abril de 2002, el Consejo de la Ciudad adoptó una posición formal con una definición y principios de sustentabilidad. Éstos fueron armados sobre una iniciativa anterior que data del año 1996, que era el Plan Estratégico para la Región Vivible (LRSP). El objetivo de este plan era mantener "vivibilidad regional" (regional liveability) y proteger el medio ambiente respecto al crecimiento anticipado en el Distrito Regional de Gran Vancouver (GVRD, 1996). Con este objetivo último, los objetivos próximos fueron los siguientes: a) aumentar las opciones de transporte; b) construir comunidades completas; c) proteger la zona verde, y d) alcanzar una zona metropolitana compacta. Estos cuatro objetivos se tradujeron en ocho indicadores claves para evaluar el desarrollo del Plan. Sin quedarse contentos, las autoridades en Vancouver han dado un paso más allá en su planificación sustentable a través del éxito obtenido en el concurso Diseño Urbano de Sistemas Sustentables de la Unión Internacional de Gas en 2003. Esta estrategia de planificación para un período de 100 años en el GRVD fue diseñada durante dos años por la empresa cities ${ }^{\text {PLUS }}$ (Planning for Long-term Urban Sustainability) y tiene sinergias con la Iniciativa de Región Sustentable actual. Esta iniciativa tiene de fondo el compromiso del Consejo de la Ciudad (2002) y el LRSP. La claridad respecto al objetivo último, con el título "Greater Vancouver 2100: A Sustainable Region in a Better World”, está expresada en la siguiente cita: "Nos comprometemos a construir una región sustentable en un mundo mejor. Nuestra región será resiliente, capaz de adaptarse a procesos predecibles de crecimiento y cambio, y también a los choques y desafíos no-predecibles que surgirán durante los próximos cien años y después. Nuestra región se mantendrá vivible, un lugar seguro y acogedor para la gente para crecer y envejecer, un lugar activo y creativo para la gente para seguir sus sueños y disfrutar de la vida” (GRVD, 1996).

Para alcanzar este objetivo, refiere en el párrafo siguiente "la visión", se considera la renovación de potenciales humanos, recursos naturales y energía, también el valor de compasión, buena gestión (good stewardship) de los recursos naturales y vínculos con otras comunidades orientándose hacia la sustentabilidad, la resiliencia y la vivibilidad.

Los cuatro puntos que se pueden comentar respecto a estos dos casos son los siguientes. El primero es el referido a la orientación. El caso de Bilbao enfatiza claramente la orientación económica de la ciudad para crear un futuro que contraste con su tradición industrial y de puerto. El caso de Vancouver destaca los valores antropocéntricos de "vivibilidad" por compasión y good stewardship de la base de recursos; en este sentido, es guiado por los principios de la sustentabilidad. Esto se relaciona con el segundo punto, de objetivos últimos y próximos. En el caso de Bilbao, parece que existe una confusión entre ellos. La "ciudad de conocimiento e innovación" es un vehículo para un desarrollo más armónico y para la cohesión social y no viceversa. El caso de Vancouver es más explícito según este objetivo último en su visión, mientras que las estrategias económicas son vehículos para este fin.

El tercer punto se relaciona con la temporalidad. En tanto la experiencia de Bilbao intenta reorientar la ciudad por su economía y el diseño urbano principalmente en el corto plazo de diez años, la experiencia de Vancouver muestra una consolidación de los principios de sustentabilidad dentro de los objetivos de su planificación urbana, por la LRPS (evaluada anualmente) $\mathrm{y}$-aunque no ha sido incorporada todavía- la iniciativa de 100 años de cities ${ }^{\text {PLUS }}$. Aunque se puede argumentar que Vancouver tiene un legado de involucramiento en la planificación urbana progresiva ${ }^{10}$, y que su base económica ha

10 La primera conferencia de Habitat tuvo lugar en Vancouver en 1976. El LRPS ganó el premio Dubai para "Mejores Prácticas en Asentamientos Humanos" en el 2002. El acontecimiento reciente más importante en este sentido fue el Foro Urbano Mundial en 2006. 
sido más sólida que la de Bilbao, sugiero que la planificación estratégica sustentable no es exclusiva de las ciudades "más" desarrolladas. Por el contrario, la planificación estratégica sustentable apunta al logro de dicha condición. En el corazón del pensamiento de desarrollo sustentable surge el concepto de la equidad intergeneracional. Sin este concepto en el centro de la planificación urbana de manera explícita, es difícil establecer cuáles de las intervenciones realizadas en una ciudad garantizan que las futuras generaciones sean capaces de satisfacer sus propias necesidades. Es un tema serio, en que no hay lugar para la respuesta insatisfactoria -"se puede importar lo que falta"-, debido a las restricciones que se producen cuando esta situación se generaliza abarcando un creciente número y tamaño de ciudades en el mundo $^{11}$.

El último punto es el referente a la participación. Sin el contexto de la necesidad que las ciudades generen inversiones - públicas y privadas- para la revitalización constante de las estrategias de desarrollo urbano, los peligros (oportunidades para otros) en entender la planificación estratégica como un ejercicio de marketing urbano están claramente relacionados con la participación. El propósito del marketing urbano debe ser promover inversiones, turismo y comunicar una identidad en transformación. Sin embargo, no debe ser un empuje principal en la planificación estratégica debido a su énfasis en fuerzas exógenas, más que fuerzas endógenas que son parte de las raíces de la planificación sustentable. Esto cambia la orientación en la participación. Una vez más, no se debe confundir la relación entre objetivos últimos y próximos. Por ejemplo, el marketing urbano es una medida de corto plazo que puede tener impactos fuertes de corto plazo: por ejemplo, el programa europeo de Ciudad de Cultura, o iniciativas urbanas individuales como la de "Joburg" de la Ciudad de Johannesburgo desde 2002, o la asociación de ciudades con eventos claves como postulaciones para ser sede de los Juegos Olímpicos o la Copa Mundial o, más específicamente, el carnaval de Río o el Oktoberfest de Munich. Son programas viables dentro de un formato de planificación

11 El comercio en agua y petróleo revela los aspectos geopolíticos de la escasez contemporánea. Esta dimensión acompaña también la escasez económica (limitaciones por precio) y la física (falta de reservas). estratégica sustentable; sin embargo, no son fines en sí mismos debido a la conexión que establecen con el mejoramiento amplio de calidad de vida local y good stewardship de los recursos naturales que, tal vez, no son explícitos.

Para aterrizar esta discusión, las implicaciones de los puntos anotados serían consideradas en el contexto de Santiago de Chile. Actualmente, la ciudad no está guiada por lo que se puede definir como planificación estratégica sustentable, debido a que el instrumento más apropiado de la autoridad competente -la Estrategia de Desarrollo Regional (2006-2010) es ambivalente en su trato de la sustentabilidad. Para ser más específico, la sustentabilidad es considerada como sustentabilidad ambiental. En el contexto del espectro que va desde la sustentabilidad débil hasta la sustentabilidad fuerte, esta construcción discursiva es muy relevante para las intervenciones urbanas que resulten.

\section{Santiago de Chile: ¿hacia la sustentabilidad?}

En el caso de Santiago, es difícil ver la consistencia en términos de la implementación de la agenda de desarrollo sustentable que surgió por los Principios de Río y el Programa 21 de 1992. Aunque Santiago jugó un rol en la promoción de la necesidad de estimular las actividades de las autoridades locales en la planificación sustentable a nivel local a través de la Carta de Nuñoa, firmada en octubre de 2002 por representantes municipales latinoamericanos en asociación con ICLEI, la planificación estratégica sustentable ha estado ausente. Más bien, las iniciativas han sido aisladas, a menudo meramente ambientales en su orientación, poco entendidas en un contexto mayor y débilmente apoyadas a nivel nacional.

A nivel nacional, los gobiernos de la Concertación, en el poder desde el año 1990, han demostrado su apoyo para el desarrollo sustentable en foros internacionales, pero han sido débiles en traducirlo en iniciativas a nivel regional y local. La creación de la Comisión de Desarrollo Sustentable en 1998, y la construcción de una Política Ambiental para el Desarrollo Sustentable el mismo año, deberían haber sugerido qué cambios significativos hacia la sustentabilidad estaban en proceso. Sin embargo, no ha sido así. La 
mayor parte de las iniciativas que han mejorado la sustentabilidad metropolitana han sido generadas por intervenciones sectoriales, con poca o nada coordinación por parte de las entidades responsables: CONAMA y la Secretaría General de la Presidencia, que son las instituciones responsables para las actividades de la Comisión de Desarrollo Sustentable ${ }^{12}$.

Cuando el desarrollo sustentable se ha traducido en el contexto urbano dentro de la Región Metropolitana, donde vive más del $40 \%$ de la población nacional y se genera más del $43 \%$ del PIB nacional (INE, 2005), esta situación se ve agravada. Tanto la inactividad de las entidades pertinentes como la competencia que se produce entre instituciones nacionales (la intervención directa de los ministerios en particular, incluyendo la presidencia), entidades regionales (secretarías ministeriales y gobierno regional) y administraciones locales (las 6 provincias y las 52 municipalidades con sus alcaldes, consejeros y profesionales respectivos) tienen una cuota de responsabilidad en esta situación. A esta complejidad en el sector público, se puede agregar el rango de actores del sector privado y la sociedad civil que operan con objetivos únicos y múltiples, desde PYMEs a empresas transnacionales, iglesias y ONGs. Es dentro de este tejido de institucionalidad que uno se enfrenta a la crisis de gobernanza para la promoción de la sustentabilidad ${ }^{13}$.

Debido a la diversidad de actores involucrados en una ciudad-región metropolitana, y a la complejidad del sistema urbano, hay una necesidad esencial de enfrentar el espacio geográfico y quienes viven sus vidas allí. Es este enfrentamiento el que crea

12 No es claro por qué una Comisión de Desarrollo Sustentable opera una política que constituye una estrategia nacional de sustentabilidad ambiental. Estas inconsistencias discursivas explican gran parte de la falta de claridad en sus operaciones e intervenciones.

13 A pesar de su importancia en generar un desarrollo más sustentable, el debate sobre gobernanza no es central en este artículo y es discutido en otras publicaciones. El proyecto DISCUS (Developing Institucional and Social Capacities for Urban Sustainability) de la Unión Europea, financiado dentro del Quinto Programa Marco de la Comisión Europea 1998-2002, es un ejemplo de un proyecto internacional de mayor escala enfocado en las dimensiones institucionales de la sustentabilidad urbana, aplicado en cuarenta ciudades europeas (véase Evans et al., 2004). el espacio para la planificación estratégica sustentable. Se discute aquí, a la luz de los temas mencionados anteriormente, sobre el instrumento territorial que tiene mayor potencial para crear una estrategia que fortalezca la sustentabilidad de la Región Metropolitana de Santiago: la Estrategia de Desarrollo Regional. Desde 1990, la Estrategia ha pasado por distintas fases. El documento más significativo para las necesidades del desarrollo sustentable es el que coincide con el desarrollo del pensamiento sobre sustentabilidad en el gobierno: la Política Ambiental de Desarrollo Sustentable de 1998, con sus fundamentos de: mejoramiento en la calidad de vida ${ }^{14}$, complementariedad entre el desarrollo socioeconómico y la sustentabilidad ambiental, y equidad social y superación de la pobreza.

Se podría haber esperado que la Estrategia de Desarrollo Regional de 2000-2006 fuera el vehículo perfecto para implementar la política nacional a nivel metropolitano. También debiera haber integrado las Metas del Milenio, firmadas en Nueva York en 2000, pero acordado en principio desde la Cumbre sobre Desarrollo Social en Copenhague en 1995. La realización del desafío de la integración de políticas en el tema ambiental de las Metas hubiera sido un pivote relevante y de buen momento de la Estrategia, pero no fue el caso ${ }^{15}$. De hecho, hasta el mensaje presidencial de la Presidenta Bachelet en mayo 2006 (Bachelet, 2006) enfatizó su ausencia: faltaba una perspectiva integrada entre la mayoría de los políticos de los gobiernos nacionales y regionales. El punto - para muchos observadores trabajando desde perspectivas multidisciplinares, y para planificadores urbanos y regionales en particular-parece muy obvio debido a las tendencias internacionales hacia la planificación. Pide una respuesta frente a por qué no había sido un tema central en la planificación

14 La Ley de Bases del Medio Ambiente define el concepto de desarrollo sustentable como "el proceso de mejoramiento sostenido y equitativo de la calidad de vida de las personas, fundado en medidas apropiadas de conservación y protección del medio ambiente, de manera de no comprometer las expectativas de las generaciones futuras" (artículo 2g) (CONAMA, 1998).

15 En el contexto del Informe GEO para Santiago del programa GEO de PNUMA, se puede ver que las evaluaciones ambientales de las áreas urbanas están, de facto, orientándose hacia evaluaciones de sustentabilidad (véase PNUMA/IEUT, 2004). 
Cuadro 3. Principios de Smart Growth y de Diseño.

\begin{tabular}{|c|c|}
\hline Los Principios de Smart Growth & $\begin{array}{l}\text { Los Principios de Hannover: } \\
\text { Diseño para la Sustentabilidad }\end{array}$ \\
\hline Usos de suelos mixtos & $\begin{array}{l}\text { Insistir en los derechos de la humanidad y } \\
\text { la naturaleza de co-existir }\end{array}$ \\
\hline Aprovechar el diseño de edificación compacta & Reconocer su interdependencia \\
\hline $\begin{array}{l}\text { Crear una variedad de oportunidades y opciones } \\
\text { de vivienda }\end{array}$ & Respetar las relaciones entre espíritu y materia \\
\hline Crear barrios caminables & $\begin{array}{l}\text { Aceptar la responsabilidad para las consecuencias } \\
\text { del diseño }\end{array}$ \\
\hline $\begin{array}{l}\text { Promover comunidades distintivas y atractivas con } \\
\text { un sentido fuerte de lugar }\end{array}$ & Crear objetos seguros de valor de largo plazo \\
\hline $\begin{array}{l}\text { Preservar los espacios abiertos, suelo agrícola, paisajes } \\
\text { naturales y áreas ambientales críticas }\end{array}$ & Eliminar el concepto de residuos \\
\hline $\begin{array}{l}\text { Fortalecer y dirigir el desarrollo hacia comunidades } \\
\text { existentes }\end{array}$ & Depender sobre los flujos de energía naturales \\
\hline Ofrecer una variedad de opciones de transporte & Entender las limitaciones del diseño \\
\hline $\begin{array}{l}\text { Hacer decisiones sobre el desarrollo más predecibles, } \\
\text { justas y efectivas }\end{array}$ & $\begin{array}{l}\text { Buscar el mejoramiento constante en compartir } \\
\text { conocimiento }\end{array}$ \\
\hline $\begin{array}{l}\text { Promover la colaboración comunitaria y } \\
\text { de stakeholders en las decisiones de desarrollo }\end{array}$ & - \\
\hline
\end{tabular}

Fuentes: Porter (2002), McDonough et al. (2002).

bajo la democracia chilena al menos desde el año 1992: "Queremos ciudades bellas, amables, equitativas. Esto supone una política urbana moderna, con sentido del territorio. Supone fortalecer la legislación e incorporar las variables ambientales y de integración social en los instrumentos de planificación territorial" (énfasis del autor).

Aunque la Estrategia 2000-2006 se vería actualizada con un plan para 2006-2010, desarrollado por el gobierno regional con la Pontificia Universidad Católica de Chile, fue la estrategia original la que fue objeto de estos comentarios debido a que fue ella, teóricamente, el mapa o blueprint para el desarrollo regional durante un período de transformación profunda que incluía la introducción de un sistema de transporte público masivo y coordinado (Transantiago), varias iniciativas de infraestructura concesionadas, preocupaciones frente a la seguridad ciudadana (incluyendo intervenciones de mayor envergadura en algunos barrios marginados), proyectos inmobiliarios privados y de vivienda social y una tendencia de crecimiento fuerte en la economía regional (véase De Mattos et al., 2004; Galetovic, 2006).

Hay dos reflexiones principales que realizar. La primera es hasta qué punto la Estrategia es, de hecho, percibida como el blueprint para la planificación estratégica en la Región Metropolitana. La se- gunda es la naturaleza de las iniciativas incluidas en la Estrategia y cómo se relacionan a lo que se puede calificar como un fortalecimiento de la sustentabilidad urbana.

Como todas las ciudades, se puede decir que Santiago es una ciudad sustentable. Es una aserción que requiere una falsación popperiana para negarla; sin embargo, es más interesante tratar de evaluar el grado de sustentabilidad y la orientación de la transformación: un fortalecimiento o un debilitamiento de la sustentabilidad urbana, además de la velocidad de esta transformación. No es un ejercicio fácil debido a la falta de métodos de evaluación acordados, a pesar de la existencia y el uso de varios otros, incluyendo la Evaluación Ambiental Estratégica (EAE), Gestión Ambiental Integrada, Evaluación Estratégica de Impactos y herramientas más específicas tales como la Evaluación de Ciclos de Vida, Factor 4, Factor 10, The Natural Step, huella ecológica, análisis de flujos de materiales o de recursos, indicador de progreso genuino (GPI) y el índice de bienestar económico sustentable (IBES) (véase Ravetz, 2000a y 2000b; Robèrt et al., 2000 y 2002; Mitchell, 2004; Brandon y Lombardi, 2005).

Es evidente que se hace necesario complementar los métodos de evaluación del desarrollo urbano existentes con estos nuevos métodos que brindan una perspectiva sobre aspectos de la sustentabilidad den- 
tro de un marco de sistemas. Eso puede incluir no solamente los métodos de evaluación, sino también principios de planificación, como los empleados en Estados Unidos con la iniciativa Smart Growth, en Australia con las Claves para el Diseño Urbano Sustentable del Estado de Victoria (Parliament of Victoria, 2004) y la Estrategia de Sustentabilidad de Western Australia (2003), y a nivel más micro, los principios de diseño sustentable acordados en Hannover en la EXPO 2000 y la certificación de construcciones como LEED (Leadership in Energy and Environmental Design) en Estados Unidos y BREAM (Building Research Establishment Environmental Assessment Method) en el Reino Unido.

Las formas en que la planificación regional estratégica más autónoma ha sido adoptada en Chile datan de principios de los ' 90 . Antes de este período, la planificación estratégica estaba centralizada en organismos a nivel nacional, en particular ODEPLAN y sus entidades regionales activas (desde 1974), las Secretarías de Planificación y Coordinación (SECPLACs). En 1990, ODEPLAN se convirtió en MIDEPLAN y asumía un estatus ministerial. El seguimiento de la planificación de desarrollo regional a principios de los ' 90 refleja a una escala menor lo que ocurre en el período de los '70, cuando la planificación urbana y regional era más fuerte como concepto y práctica ${ }^{16}$.

En el caso de la Región Metropolitana, la Estrategia de Desarrollo 1995-2000 tuvo la intención de integrar distintos componentes de un ejercicio de planificación estratégica (Lira, 1990): la visión, el diagnóstico (FODA), objetivos y metas, diseño y formulación de políticas y programas y su monitoreo y evaluación.

16 Se pueden citar varios autores influyentes durante este período, como Friedmann (1969), Stohr (1972), Rondinelli (1975) y Bosier (1981). Gran parte de este trabajo, con su amplio alcance y responsabilidad pública, es clave para la perspectiva neoestructuralista de la planificación estratégica metropolitana orientada hacia la sustentabilidad. Los desafíos, tales como la descentralización, la coordinación de actores y la participación de los mismos, siguen en el nuevo siglo. Tal vez la diferencia clave es el fuerte core económico de esta perspectiva de planificación regional de los años '70 (véase ODEPLAN, 1978).
Una revisión crítica de la Estrategia sugiere que, a pesar de su acercamiento a la sustentabilidad en términos de la inclusión de un rango de factores con ejes estratégicos (con acciones, programas y proyectos asociados) orientados hacia el desarrollo espacial, productivo, social, infraestructural y ambiental, éstos no fueron integrados dentro de un marco de la sustentabilidad. Es evidente que el informe Brundtland y el Programa 21 no son guías para la Estrategia, y varios de los requisitos -como la participación amplia y un horizonte intergeneracionalestán ausentes. De todas maneras, la Estrategia -como carta de navegación ${ }^{17}$ para el Área Metropolitana en el corto plazo- tiene una visión clara: "Mejorar la calidad de vida de los habitantes metropolitanos a través de la construcción de un desarrollo armónico, coherente, integrado y más humano de nuestras ciudades y de nuestra región" (Gobierno Regional Metropolitano, 1995: 27).

La declaración favorece lo que Acselrad (1999) define como el discurso de "calidad de vida" de la sustentabilidad, el cual contrasta con lo "técnicomaterial" o el discurso de "legitimación de política urbana”. No obstante, el detalle respecto a objetivos se mueve rápidamente en la dirección de estos discursos alternativos. También es el caso con la Estrategia 2000-2006 con su visión más extendida, y tal vez menos concisa (aunque similarmente orientada): "Promover la transformación de sus habitantes en ciudadanos solidarios, recuperar la identidad regional sobre la base de su diversidad cultural y crecer en equilibrio, avanzando en la construcción de un mejor territorio para vivir. Desarrollar mejores usos de las infraestructuras y tecnologías, fomentar el acceso a oportunidades competitivas que fortalecen un compromiso económico sostenible, ambientalmente sustentable y socialmente integrado, así como la gestión responsable y articulada entre las autoridades regionales, provinciales, comunales y sectoriales de Santiago región de Chile y Santiago región del mundo" (Gobierno de Chile, 2000: 12).

En este último caso, la visión era acompañada con cinco áreas temáticas: ciudadanía, sustenta-

17 Este concepto de estrategia -como carta de navegación- era utilizado por el entonces Intendente de la Región Metropolitana, Sergio Galilea, en su introducción a la Estrategia 2000-2006. 
bilidad espacial y ambiental, competitividad, modernización de la gestión pública regional y promoción de identidades y culturas regionales.

Cuando uno examina el uso y la interpretación del concepto de sustentabilidad en los dos casos, es posible ver que la sustentabilidad no es central a sus agendas. Ello a pesar del uso de los distintos mecanismos del pensamiento de la sustentabilidad, como la incorporación de los aspectos institucionales, sociales, ambientales y económicos del proceso de desarrollo, unida a una visión que incorpora un ámbito similar a la del desarrollo sustentable como está definido en el informe Brundtland ${ }^{18}$, en términos del mejoramiento en calidad de vida y espacios para vivir. En la Estrategia 1995-2000, existe una clara confusión en términos de los objetivos últimos y próximos. En la determinación de 16 factores estratégicos, basado en un análisis FODA, el factor 5 revela la necesidad de un "desarrollo socialmente, territorialmente y ambientalmente armónico", y factor 11 , el "uso óptimo de recursos naturales". No obstante, la integración de estos factores, en términos de jerarquía y traslapes queda subdesarrollado. Son tres los ejes o "ideas centrales" que surgen de estos factores (Gobierno Regional Metropolitano, 1995: 28):

El gobierno regional ha tomado responsabilidad para la gestión del desarrollo de la Región, articulando la actividad regional de los sectores público y privado, para alcanzar objetivos consensuados de desarrollo.

- La Región Metropolitana se ha convertido en un espacio para vivir que es moderno y ofrece oportunidades, basado en el uso racional de recursos que contiene.

- $\quad$ La ciudad de Santiago es un espacio vital que ofrece una alta calidad de vida a todos sus habitantes.

Es en el área de los criterios que orientan la gestión regional que los principios de la sustentabilidad -postulados en los requisitos para el desarrollo sus-

18 El desarrollo sustentable es "el desarrollo que satisface las necesidades de la presente generación sin comprometer la capacidad de las futuras generaciones para que satisfagan sus propias necesidades". tentable identificados en el informe Brundtlandson más evidentes. Se destaca que el desarrollo está basado en la equidad y la distribución equitativa de oportunidades; desarrollo espacial equilibrado en armonía con el medio ambiente; desarrollo económico que corresponde al modelo nacional; y administración y gestión pública que opera en términos de eficiencia, racionalidad y complementariedad.

La Estrategia 2000-2006 sigue esta línea, pero hay poco desarrollo del concepto de sustentabilidad. $\mathrm{Al}$ contrario, hay una clara falta de comprensión de éste, o un abuso del término en la precisión y construcción de la categoría "sustentabilidad ambiental". Es dentro de esta área que el concepto está manejado, por lo que no aparece en otras áreas de la estrategia, por ejemplo interrelacionando explícitamente criterios sociales y económicos. Dentro de este eje de la estrategia, hay una colección de intervenciones que se puede definir como "infraestructural" la mayoría de las veces. Es significativo destacar que este eje concentra la mayor parte de las inversiones de la Estrategia y los proyectos más emblemáticos de los años ' $90^{19}$. Incluye el plan maestro para el tratamiento de efluentes domésticas, el sistema de autopistas urbanas y el sistema de colectores de aguas lluvias. El grado de sustentabilidad asumido por cada proyecto no queda claro.

Parece que la construcción de la "sustentabilidad ambiental" en este sentido está altamente relacionada con lo que se puede definir como la modernización ecológica (Christoff, 1996; Hajer, 1997). Esencialmente, la modernización ecológica promueve eco-eficiencias y "soluciones" tecnológicas hacia "problemas" ambientales. El informe Brundtland y el Programa 21 comparten fuertes rasgos de la modernización ecológica, que ha sido criticada por algunos como demasiado "optimista tecnológicamente". Debido a su falta de crítica hacia el modelo de desarrollo contemporáneo y sus raíces capitalistas, los críticos enfatizan que hay limitaciones

19 Un 48\% de la inversión total, indicado en la Estrategia, era dirigido hacia el área de Sustentabilidad Territorial Ambiental. Ésta incluye los proyectos relacionados con la construcción de autopistas urbanas, el plan de descontaminación atmosférica, la gestión de residuos líquidos y sólidos, la renovación urbana, un parque comercial (ENEA) y el plan de transporte para la ciudad (Gobierno de Chile, 2000). 
internas en términos de un aumento en bienestar o calidad de vida, en un horizonte intergeneracional (véase Pepper, 1993; Finger y Chatterjee, 1994; Larraín, 1999). La inclusión de autopistas urbanas dentro de esta categoría de la "sustentabilidad ambiental" es tal vez un abuso del término. Muchas perspectivas contemporáneas sobre la planificación, como Smart Growth y Slow Cities, apuntan a la necesidad de "descarbonizar" las economías, promover sistemas de transporte público, desincentivar el uso de vehículos privados a través del "cobro por uso" y otros instrumentos económicos, con una mirada más allá de la construcción de la "ciudad del automóvil", tan predominante en el pensamiento de planificación durante la segunda mitad del siglo veinte -esta intervención parece ser una reliquia de los años '60, el momento cuando era discutida por primera vez.

Asociados con estos proyectos de infraestructura está la temporalidad de las inversiones, que por lo común aparecen totalmente diferenciadas de la estrategia de planificación desde la cual nacen. Mientras que estos proyectos están diseñados para un período de a lo menos veinte años, la estrategia de planificación tiene una temporalidad de seis años. Por lo mismo, es claro que la estrategia se orienta precisamente a construir la ciudad intergeneracional, mientras su propio horizonte de tiempo está dado por la administración política, en este caso el período presidencial de Ricardo Lagos (2000-2006). En la misma línea, el período 2006-2010 más probablemente será dominado por varios proyectos urbanos denominados Bicentenarios, por ejemplo el rediseño de la proyección hacia el sur desde el palacio presidencial (incorporando un centro cultural) y el reciclaje del sitio del aeropuerto de Cerrillos. Estos proyectos son diseñados a muy largo plazo. Se puede deducir entonces que la planificación estratégica concurrente incluye un rango de objetivos de corto, mediano y largo plazo. La consecuencia es que las estrategias orientadoras desde el año 2000 han influido en la toma de decisiones dentro de un período administrativo, mientras que han dejado una huella significativa en el paisaje urbano que es definitivamente intergeneracional.

Respecto a los temas claves relacionados con la planificación estratégica sustentable-compromiso orientación, objetivos últimos y próximos, tempo- ralidad, y participación- se puede observar lo siguiente en el contexto de Santiago. El compromiso presente en el proceso de planificación regional surge de la necesidad de orientar mejor e integrar diversos elementos del desarrollo regional. Este compromiso no es el de la sustentabilidad, sino un conjunto de aspectos percibidos como positivos para el desarrollo, entre ellos la "sustentabilidad ambiental". Aunque la orientación principal está enfocada en un mejoramiento de la calidad de vida, la confusión entre distintos objetivos se traduce en que la relación entre actividades y ejes estratégicos y la realización de un mejoramiento en la calidad de vida no es clara. El período de implementación es demasiado corto para ser una estrategia intergeneracional; corresponde más bien a un plan de operaciones administrativas. Tal vez las debilidades más significativas -a pesar de la falta de coherencia en torno a la sustentabilidad en generalsean la participación en la creación del documento y su realización. La falta de participación amplia en las estrategias, la falta de un consenso más allá de uno "técnico-material" y la débil inclusión de la Estrategia en otros instrumentos, como los planes de desarrollo comunal y políticas públicas sectoriales, han llevado a que las estrategias se conviertan, hasta cierto punto, en una carta de navegación para el gobierno regional pero no para la multitud de otros actores que transforman la ciudad a través de sus actividades cotidianas.

Frente a estas críticas, se puede argumentar que la sustentabilidad no fue central en el proceso de planificación. Por ende, no sorprende que éste no cumpla con los criterios de la sustentabilidad. Sin embargo, la política nacional de sustentabilidad (1998) y otras iniciativas y acciones acordadas en foros internacionales (Río de Janeiro en 1992, Santa Cruz de la Sierra en 1996, Johannesburgo en 2002, entre otros) y nacionales, con una institucionalidad relevante (la Comisión de Desarrollo Sustentable), deben llegar a ser relevantes en los procesos de planificación.

\section{La planificación estratégica para la sustentabilidad}

El caso de Santiago de Chile, contrarrestado con las experiencias de Bilbao, Vancouver, Londres y otros, revela que la última década ha sido testigo de 
un crecimiento fuerte en la planificación estratégica. Aunque los procesos y los contenidos de estos procesos de planificación varían, es claro que el desarrollo sustentable ha estado presente como concepto en su formulación; es más o menos explícito en cada caso. No obstante, el hecho de que exista y muestre los traslapes con el instrumento de planificación urbana estratégica -por ejemplo participación, horizontes de largo plazo, toma de decisiones en forma integrada, institucionalidad-sugiere que tienen mucho en común. Tal vez el traslape más destacable es el pensamiento intergeneracional o pensamiento de futuros (véase, por ejemplo, Hjorth y Bagheri, 2005; Hughes y Johnston, 2005). La planificación de ciudades con horizonte de a los menos veinte años se contrasta con las experiencias más tradicionales de planificación de cinco o diez años que sugiere una asociación más cercana a las económicas de comando soviéticas que a las sociedades democráticas del siglo XXI.

El concepto de equidad es también relevante. En el informe Brundtland, la equidad intergeneracional era el buzz-phrase que condicionaba la lectura de los contenidos del informe. La inclusión social, la participación, el aprendizaje social, las redes y el diálogo son todos modus operandi contemporáneos para alcanzar futuros urbanos más equitativos y, por ende, espacios urbanos más democráticos. Es solamente cuando este concepto está integrado en las conceptualizaciones contemporáneas de la ciudad del futuro que la ciudad moderna, competitiva, generalmente conflictiva (tanto intra como interurbana) puede ser transformada en la ciudad más cooperativa y colaborativa (inter e intraurbana). A través del énfasis en los objetivos compartidos, las responsabilidades compartidas y las oportunidades compartidas, debe ser posible caminar hacia un fortalecimiento de la sustentabilidad urbana para mejorar las calidades de vida actuales y futuras. La planificación estratégica tiene un rol clave en esta transformación.

La necesidad para el pensamiento de largo plazo y la generación de visiones compartidas para las ciudades es un sine qua non de la planificación contemporánea. En el mismo sentido que la planificación estratégica es central para las actividades del sector privado, también lo es para las autoridades públicas que buscan evitar conflictos y promover participa- ción y consenso vis-à-vis formas y gestión urbanas. El backcasting desde objetivos futuros deseados hacia la toma de decisiones de mediano y corto plazo parece ser un enfoque planificado y normativo para equilibrar las complejidades de las demandas, riesgos y situaciones diversas que se presentan en los urbanos modernos. Esto contrasta con los roles facilitadores y regulatorios de muchas administraciones urbanas que perciben sus roles como reacciones a las intervenciones de un rango amplio de actores, a través de la fiscalización de instrumentos legales. Una posición más proactiva respecto a los objetivos de calidad de vida en el largo plazo, y cómo se traducen en reestructurar paisajes y prácticas, surge como la tendencia contemporánea para las autoridades metropolitanas que buscan evitar las experiencias costosas y traumáticas de los conflictos urbanos, mientras al mismo tiempo toman en cuenta los deseos de una base amplia para los futuros urbanos de calidad de vida.

Sugerir que este cambio en pensamiento es una forma más fácil de conceptualizar y gestionar los espacios urbanos sería erróneo. No obstante, ofrece un campo de acción que complementa actividades existentes y brinda proyecciones y escenarios de largo plazo sobre los cuales la gente puede contestar las estrategias y opciones del desarrollo urbano, y alcanzar un consenso participativo que sea preferible a una "solución" técnica de "expertos" o una salida políticamente oligárquica a los dilemas de estilos de vida urbanos del siglo XXI.

La planificación estratégica sustentable es un instrumento o proceso que puede apoyar esta orientación. En distintos sentidos, es un enfoque radical hacia la planificación metropolitana que se mueve más allá del ámbito técnico para incluir múltiples consideraciones políticas y éticas, como la de equidad. La cita que sigue capta esta orientación muy bien, destacando que las ciudades son espacios en que la gente busca satisfacer sus necesidades y realizar sus aspiraciones. Es el mejoramiento en la calidad de vida de los residentes urbanos el objetivo último de la planificación urbana, y los principios de la sustentabilidad son centrales para su logro, a través de un desarrollo equilibrado, armónico, integrado y equitativo: "En mi opinión, cuando nos referimos al desarrollo sustentable, hablamos fundamentalmente de una nueva idea de desarrollo, y nos acercamos 
cada vez más al concepto de desarrollo humano, que cada día tiene más peso en la humanidad. Cuando hablamos poniendo énfasis en lo ambiental, nos referimos a un desarrollo que tenga como centro el ser humano y su calidad de vida" (Cámara de Diputados, 1997: 8).

La cita anterior corresponde al ex presidente de la Comisión de Recursos Naturales, Bienes Nacionales y Medio Ambiente de la Cámara de Diputados. En 1997, antes de la formación de la Comisión de Desarrollo Sustentable, una sesión de la Comisión fue organizada en torno a "un diálogo sobre el desarrollo sustentable". La introducción del presidente de la Comisión era muy apropiada en términos de la necesidad de entender la sustentabilidad como un cambio importante en como apreciamos el desarrollo contemporáneo. Una década más tarde, Victor Barrueto es ahora Intendente de la Región Metropolitana y responsable para el proceso de planificación estratégica para la Región desde 2010. Es precisamente esta perspectiva sobre sustentabilidad la que debe ser central en tal proceso.

\section{Referencias bibliográficas}

Ache, P. (2000). "Vision and creativity-challenge for city regions". Futures, 32: 435-449.

Asia and Pacific Leadership Forum (2004). "Hong Kong Declaration on sustainable development for cities". http://www.un.org/esa/sustdev/csd/ csd12/HK_declaration.pdf.

Acselrad, H. (1999). "Sustentabilidad y ciudad". EURE Revista Latinoamericana de Estudios Urbano Regionales, 25, 74: 36-46.

Allen, A. et al. (2002). Sustainable Urbanisation: Bridging the green and blue agendas. London: DPU/DFID.

Bachelet, M. (2006). "Discurso 21 de Mayo 2006" (Mensaje Presidencial). http:// w w w. presiden cia.cl/vi e w / viewArticulo.asp?idArticulo=3229\&tipo=Discursos \&seccion=Discursos.

Bilbao Metrópoli-30 (2006). "Bilbao as a global city: New strategic challenge for 2010"; "Bilbao 2010: La Estratégia”. http://www.bm30.es/ homeage9_uk.html.

Boisier, S. (1981). Planning a system of regions. Santiago: ILPES.
Brandon, P. y P. Lombardi (2005). Evaluating sustainable development in the built environment. Oxford: Blackwell.

Bryson, J. y W. Roering (1993). "Applying private sector strategic planning in the public sector". Lincoln Institute of Land Policy (ed.), Strategic Urban Management: Resource Manual. Cambridge, MA.: LILP, 15-34.

Cámara de Diputados (1993). Diálogo para el desarrollo sustentable (redacción de sesiones, Comisión de Recursos Naturales, Bienes Nacionales y Medio Ambiente). Valparaíso: Congreso Nacional.

Campbell, S. (2005). "Green cities, growing cities, just cities? Urban planning and the contradictions of sustainable development". Campbell, S. y S. Fainstein (eds.), Readings in planning theory. Oxford: Blackwell, 435-458.

Comisión Nacional del Medio Ambiente (1998). Una politica ambiental para el desarrollo sustentable. Santiago: CONAMA.

CSD/ICLEI (2002). Second Local Agenda 21 survey. Background paper 15. New York: UN Department of Social and Economic Affairs.

Commission for Sustainable Development (2002). "Plan of implementation of the world summit on sustainable development". http:// www.un.org/esa/sustdev/documents/ W S S D_P O I _ P D / Englis h/ WSSD_PlanImpl.pdf.

Christoff, P. (1996). "Ecological modernisation, ecological modernities". Environmental Politics, 5, $3: 476-500$.

De Mattos, C. et al. (eds.) (2004). Santiago en la globalización: ¿una nueva ciudad? Santiago: SUR/EureLibros.

Escobar, A. (1995). Encountering development: The making and unmaking of the Third World. New Jersey: Princeton University Press.

Evans, B. et al. (2004) Governing Sustainable Cities. London: Earthscan.

Fernández Güell, J.M. (2000). Planificación estratégica de ciudades. Barcelona: Gustavo Gili.

Finger, M. y P. Chatterjee (1994). The Earth Brokers: Power, politics and world development. New York: Routledge.

Friedmann, J. (1969). Urban and regional development in Chile: A case study of innovative planning. Santiago: Ford Foundation. 
Galetovic, A. (ed.) (2006). Santiago: dónde estamos y hacia dónde vamos. Santiago: CEP.

Gibson, R. et al. (2005). Sustainability assessment: Criteria, processes and applications. London: Earthscan.

Gross, P. (2002). Sustentabilidad: ¿un desafio imposible? Santiago: Surambiente.

Gobierno Regional Metropolitano (1995). Estrategia de Desarrollo: construir la región es construir pais 1995-2000. Santiago: Gobierno de Chile.

Gobierno de Chile (2000). Región Metropolitana de Santiago, 2000-2006-Estrategia de Desarrollo. Santiago: Gobierno de Chile.

Greater Vancouver Regional District (1996). The Liveable Region Strategic Plan. Vancouver: GVRD.

Hajer, M. (1997). The politics of environmental discourse: Ecological modernisation and the policy process. Oxford: Oxford University Press.

Hjorth, P. y A. Bagheri (2006). "Navigating towards sustainable development: A system dynamics approach". Futures, 38: 74-92.

Hughes, B y P. Johnston (2005). "Sustainable futures: policies for global development”. Futures, 37: 813-831.

Instituto Nacional de Estadísticas (2005). "Compendio estadístico 2005”. http://www.ine.cl/ ine/canales/chile_estadistico/ compendio_estadistico/pdf/2005/3.pdf.

Larraín, S. (1999). Chile sustentable: propuesta ciudadana para el cambio. Santiago: LOM.

Lira, L. (1990). "La confección de Estrategias de Desarrollo Regional". Arenas, F. (ed.), Manual de desarrollo regional. Santiago: INC-PREDREGION-IEU, 41-68.

Macedo, J. (2004). "City profile: Curitiba”. Cities, 21, 6: 537-549.

Marcotullio, P. J. (2001). "Asian urban sustainability in the era of globalization". Habitat International, 25: 577-598.

Meadowcroft, J. (1999). "Planning for sustainable development: what can be learned from the critics?" Kenny, M. y J. Meadowcroft (eds.), Planning sustainability. London: Routledge, 1238.

McDonough et al. (2002). "The Hannover Principles: Design for sustainability". http:// www.mcdonough.com/principles.pdf.
Mitchell, G. (2004). "Forecasting urban futures: A systems analytical perspective on the development of sustainable urban regions". Purvis, M. y A. Grainger (eds.), Exploring sustainable development: Geographical perspectives. London: Earthscan, 99-127.

Organisation of American States (1996). "Plan de acción para el desarrollo sostenible de las Américas". http://www.science.oas.org/espanol/ stacruz_plan.htm.

ODEPLAN (1978). Experiencia de planificación regional en Chile. Santiago: ODEPLAN.

Parliament of Victoria (Australia) (2004). Inquiry into sustainable urban design for new communities in outer suburban areas: Final report. Melbourne: State of Victoria.

Pepper, D. (1993). Ecosocialism: From deep ecology to social justice. London: Routledge.

PNUMA/IEUT (2004). Informe GEO Santiago de Chile 2003. Santiago: EureLibros.

Porter, D. (2002). Making Smart Growth work. Washington, D.C.: ULI.

ProMadrid S.A. (2003). Madrid Futuro: plan estratégico de Madrid. Madrid: ProMadrid S.A..

Ravetz, J. (2000a). "Integrated assessment for sustainability appraisal in cities and regions". Environmental Impact Assessment Review, 20: 3164.

(2000b). City Region 2020: Integrated planning for a sustainable environment. London: Earthscan.

Robèrt, K-H et al. (2000). "Tools and concepts for sustainable development, how do they relate to a general framework for sustainable $\mathrm{d}$ evelopment, and to each other?" Journal of Cleaner Production, 8: 243-254. (2002). "Strategic sustainable development -selection, design and synergies of applied tools". Journal of Cleaner Production, 10: 197-214.

Rostow, W. W. (1960). The stages of economic growth: A non-communist manifesto. Cambridge: Cambridge University Press.

Rondinelli, D. (1975). Urban and regional development planning: policy and administration. Ithaca: Cornell University Press.

Stiglitz, J. (2002). Globalization and its discontents. New York: W.W. Norton. 
Stohr, W. (1972). El desarrollo regional en América Latina: experiencias y perspectivas. Buenos Aires: SIAP.

Swyngedouw, E. (1992). “The Mammon Quest: 'Glocalization', interspatial competition and the monetary order: The construction of new scales". Dunford, M. y G. Kafkalis (eds.), Cities and regions in the new Europe. The global-local interplay and spatial development strategies. London: Belhaven Press, 39-67.

Tedesco, L. y J. Barton (2004). The state of democracy in Latin America: Post-transitional crises in Argentina and Chile. London: Routledge.

UN (2002). "Plan of implementation of the World Summit on Sustainable Development". http:// www.ine.cl/ine/canales/chile_estadistico/ compendio_estadistico/pdf/2005/3.pdf.
(2006). “The UN Millennium Development Goals”. http://www.un.org/millenniumgoals/\#. Unsworth, R. (2004). "Making cities more sustainable: People, plans and participation”. Purvis, M. y A. Grainger (eds.), Exploring sustainable development: Geographical perspectives. London: Earthscan, 128-155.

Wallerstein, I. (1974). The modern world system. New York: Academic Press.

Winchester, L. (2005). Sustainable human settlements development in Latin America and the Caribbean. Serie Medio Ambiente y Desarrollo, 99. Santiago: CEPAL.

WCED (1997). Our Common Future. Oxford: Oxford University Press. 